\title{
Studies on $\mathrm{Ge} / \mathrm{CeO}_{2}$ thin film system using positron beam and Raman spectroscopy
}

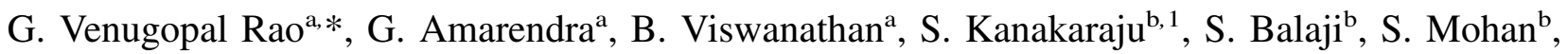 \\ A.K. Sood ${ }^{\mathrm{c}}$ \\ ${ }^{a}$ Materials Science Division, Indira Gandhi Centre for Atomic Research, Kalpakkam 603102, India \\ ${ }^{\mathrm{b}}$ Department of Instrumentation, Indian Institute of Science, Bangalore 560012, India \\ ${ }^{\mathrm{c}}$ Department of Physics, Indian Institute of Science, Bangalore 560012, India
}

Received 6 December 2000; received in revised form 20 November 2001; accepted 8 January 2002

\begin{abstract}
Positron beam and Raman spectroscopic studies are reported on a $\mathrm{Ge} / \mathrm{CeO}_{2}$ thin film system grown on a $\mathrm{Si}$ substrate. The variation of the Doppler line shape S-parameter, as a function of positron beam energy, shows a pronounced maximum corresponding to the Ge-region of as-grown film, while no equivalent feature is seen in the sample annealed at $773 \mathrm{~K}$. The Raman spectrum of the as-grown film exhibits a broad band, as opposed to a sharp peak observed for the annealed sample. Based on the correlated evidence from the two measurements, it is concluded that the Ge layer in the as-grown sample is amorphous, containing a large concentration of structural vacancies. Isochronal annealing measurements of the lineshape parameter in the temperature range of 300 to $773 \mathrm{~K}$ indicate a rather continuous transformation of amorphous Ge to the crystalline state. (C) 2002 Elsevier Science B.V. All rights reserved.
\end{abstract}

Keywords: Amorphous materials; Germanium; Positron spectroscopy; Raman scattering; Defects

\section{Introduction}

Properties of thin layer structures are very sensitive to the presence of defects, composition, crystallinity and local atomic bonding configuration, as shown by the use of interference enhanced Raman spectroscopy (IERS) [1]. Study of trilayer structures, comprising a thin semiconducting layer, a dielectric layer and a reflecting layer, has evoked interest in recent times. More amount of light can be trapped in the top thin semiconducting layer to be studied, whose thickness is less than the penetration depth of the incident light. The second layer beneath is a transparent dielectric film (normally $\mathrm{SiO}_{2}$ ), which introduces the required phase shift (hence called the phase layer) and the bottom layer is a reflector. A variety of experimental techniques are being employed to characterise the properties of these

\footnotetext{
*Corresponding author. Fax: +91-4114-480081.

E-mail address: gvenu@igcar.ernet.in (G. Venugopal Rao).

${ }^{1}$ Present address: Department of Electrical Engineering, Thornton Hall, University of Virginia, Charlottesville, VA 22903, USA.
}

heterostructures in both ex-situ and in-situ conditions. Among the optical techniques, Raman spectroscopy [2] is an excellent tool for probing the structure, degree of crystallinity, composition, strain and quality of the interface. Near-surface Raman scattering measurements performed in-situ in ultra high vacuum, have been reported [3] on Ge clusters and ultra thin amorphous films, deposited on low reflectance trilayer structures composed of $\mathrm{Al}, \mathrm{SiO}_{2}$ and a top layer of $\sim 20 \mathrm{~nm}$ of C. In this study, substantial changes in the amorphous-like Raman spectral form with decreasing film thickness indicate the increasing role of near-surface dangling bonds and bond angle disorder on the phonon density of states. In recent studies on trilayer structures [4-6], the conventionally used amorphous $\mathrm{SiO}_{2}$ has been replaced by crystalline $\mathrm{CeO}_{2}$ (fluorite structure) in order to grow crystalline $\mathrm{Ge}$ film at relatively lower substrate temperatures. Here, the dielectric phase layer of $\mathrm{CeO}_{2}$ also serves as a buffer layer. IERS study [5] of a trilayer structure of $\mathrm{Al}, \mathrm{CeO}_{2}$ and ultra thin Ge film has shown an increased red shift of the peak position and line 
broadening, with a decrease of Ge film thickness. The changes in the Raman shift as a function of film thickness, have shown that the films are compressively strained up to a thickness of $\approx 7 \mathrm{~nm}$, beyond which the strain is released. In-situ Raman monitoring [6] of ultra thin Ge films has revealed disordered surface with a large number of dangling bonds. Recently, IERS has been used to study surfactant-mediated growth of ultra thin $\mathrm{Ge}$ and $\mathrm{Si}$ films and their interfaces [7]. While Raman spectroscopy is sensitive to the crystallinity of the sample, positron annihilation spectroscopy [8] is a powerful technique with sensitivity for vacancy type defects in the fractional concentration range of $10^{-7}$ to $10^{-4}$. With the emergence of variable low energy positron beam (LEPB), selective depth profiling of nearsurface defects in thin film sample has become possible [9]. The defect-sensitivity coupled with the depth resolved capability of LEPB, facilitates rather unique characterisation of defects in thin-layered structures. The depth distribution of vacancy type defects and subsurface amorphisation in $140 \mathrm{keV}$ Ar ion irradiated $\mathrm{Si}$ have been studied [10] by a combination of LEPB, Raman and ion-channelling techniques. Studies of different states of strain relaxation and the associated defect profiles across the interface have been reported [11] in GaAs based heterostructures, which find applications in the development of quantum well lasers.

In the present paper, we report results using a combined study of Raman and positron beam spectroscopy to obtain structural and defect specific information on $\mathrm{Ge} / \mathrm{CeO}_{2}$ films deposited on $\mathrm{Si}$ substrates. The emphasis in the present study is to characterise the Ge layers in terms of crystallinity and structural defects in the asgrown state and after isochronal annealing treatment.

\section{Experimental details}

Samples of $\mathrm{Ge} / \mathrm{CeO}_{2}$ were prepared by sequentially depositing $\mathrm{Ge}$ film of $100 \mathrm{~nm}$ and $\mathrm{CeO}_{2}$ film of 100 $\mathrm{nm}$ thickness on to $\mathrm{Si}(100)$ substrates, with $\mathrm{Ge}$ being the top layer. These films were deposited by argon ion beam sputter deposition [4]. The substrate temperature was kept at room temperature $\sim 300 \mathrm{~K}$. The ceria films were sputtered at a total operating pressure of $0.02 \mathrm{~Pa}$ from a stoichiometric $\mathrm{CeO}_{2}$ target, including the oxygen partial pressure of $0.01 \mathrm{~Pa}$. The target used for the deposition of Ge film was single crystal Ge(111) of purity $99.999 \%$ and the Ge film was deposited at an argon pressure of less than $0.003 \mathrm{~Pa}$. The thickness of the film was estimated based on the calibration rate of deposition, using a Talysurf (Rank Taylor Hobson Ltd.) quartz crystal monitor. As opposed to the earlier reported studies [4-7] on very thin films (thickness $\leq 10 \mathrm{~nm}$ ), the present studies have been carried out on $\mathrm{Ge} / \mathrm{CeO}_{2}$ samples with $\mathrm{Ge}$ film thickness of $100 \mathrm{~nm}$, which is comparable with the intrinsic positron diffusion length
[9]. This will help in delineating the annihilation events occurring at the surface state and inside the Ge layer, in the LEPB study.

LEPB measurements were carried out using a magnetically guided beam system [12]. From Doppler measurements of the annihilation $511 \mathrm{keV} \gamma$-ray, a defect sensitive line shape parameter viz., S-parameter is deduced $[9,12]$. Owing to the presence of positron trapping at open volume defects, Doppler broadening curve gets narrower, yielding a higher S-parameter. Positron beam energy $E_{\mathrm{p}}$ was varied from $200 \mathrm{eV}$ to 20 $\mathrm{keV}$ in incremental steps of $500 \mathrm{eV}$, so as to probe various sample depths and S-parameter evaluated at each positron beam energy. The mean positron implantation depth $\bar{z}$ can be related to the positron beam energy $E_{\mathrm{p}}$ through the power law [9], $\bar{z}=A E_{\mathrm{p}}^{n}$. The constant $A$ is found empirically to be $A \sim 40 / \rho \mathrm{nm} / \mathrm{keV}^{n}$, where $\rho$ is the density of the material in $\mathrm{g} / \mathrm{cm}^{3}, \bar{z}$ is in $\mathrm{nm}$ and $E_{\mathrm{p}}$ is in $\mathrm{keV}$. The exponent $n$, which depends on the nature of the material and on the energy range, is found to be $\approx 1.6$ for positrons incident on several materials. The power law with $n \approx 1.6$ has been applied to the present system under study to obtain a qualitative depth calibration. By analysing the variation of S-parameter as a function of positron beam energy, information pertaining to the nature and depth distribution of defects in the sample can be obtained. LEPB measurements were made at room temperature on the as-grown and isochronally annealed samples. Annealing of samples was carried out from $300 \mathrm{~K}$ to $773 \mathrm{~K}$ in a vacuum of $10^{-6}$ torr, with a holding time of $30 \mathrm{~min}$ at each annealing step. Raman measurements were carried out at room temperature in the back-scattering configuration, using the $514.5 \mathrm{~nm}$ line of an argon-ion laser operating at a low power of $\sim 2 \mathrm{~mW}$. A Dilor X-Y spectrometer equipped with a liquid-nitrogen cooled CCD detector was used to collect the signal. Raman measurements were carried out on as-grown sample and the sample annealed at $773 \mathrm{~K}$.

\section{Results and discussion}

Fig. 1 shows the variation of normalised S-parameter as a function of positron beam energy $E_{\mathrm{p}}$ for the asgrown $\mathrm{Ge} / \mathrm{CeO}_{2}$ film and sample annealed at $773 \mathrm{~K}$. In Fig. 1, normalisation has been done with respect to the S-parameter value of the $\mathrm{Si}(100)$ substrate, which does not vary with annealing temperature. The experimental error bar for the measured S-parameter is of the order of size of the symbol used. The mean depth scale probed by positron beam is indicated on the top axis. The following are the salient features observed in Fig. 1. (a) The starting value of the S-parameter corresponding to $E_{\mathrm{p}} \approx 0$, is characteristic of the surface and this value is higher for the as-grown surface as compared with that for the annealed sample. This is indicative of defects 


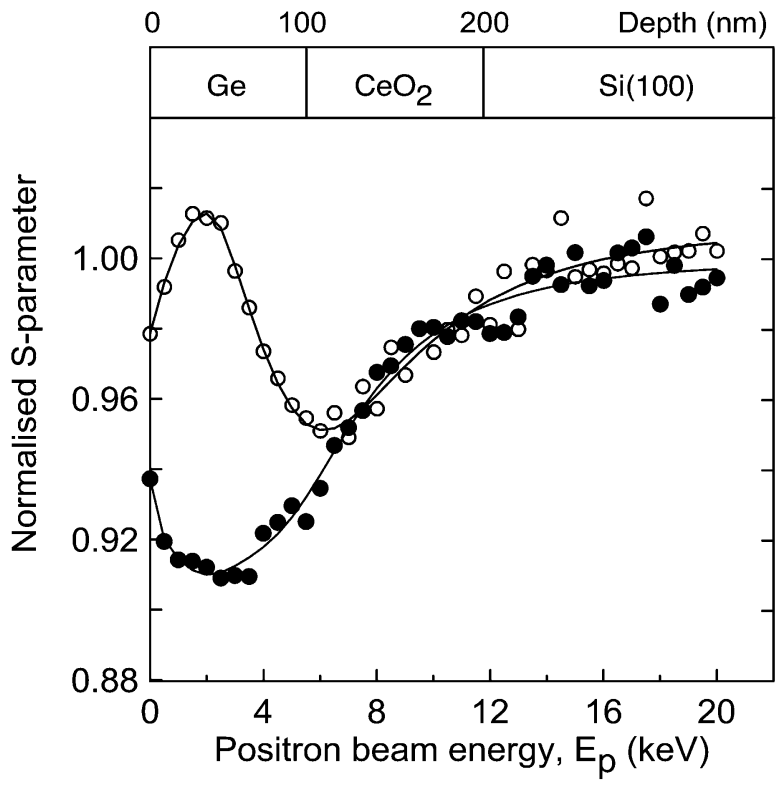

Fig. 1. Variation of the normalised line shape S-parameter as a function of positron beam energy for the as-grown $\mathrm{Ge} / \mathrm{CeO}_{2}$ film $(\bigcirc)$ and sample annealed at $773 \mathrm{~K}(\bullet)$. The solid line through the data points is the result of VEPFIT analysis. The depth scale probed by positron beam is indicated on the top axis.

associated with a large number of dangling bonds at the surface of the as-grown film. (b) As the beam energy is increased, slow positrons probe the Ge-region. For the as-grown film, the S-parameter starts increasing and exhibits a pronounced maximum at approximately $E_{\mathrm{p}}$ of $2 \mathrm{keV}$, beyond which it starts decreasing. This pronounced maximum at approximately $2 \mathrm{keV}$ is absent in the sample annealed at $773 \mathrm{~K}$. (c) The dip seen in the S-parameter of the as-grown sample at $E_{\mathrm{p}} \sim 6 \mathrm{keV}$ is associated with the interface between $\mathrm{Ge}$ and $\mathrm{CeO}_{2}$ layer. (d) At higher incident beam energies (beyond $E_{\mathrm{p}} \sim 6 \mathrm{keV}$ ), positron probes deeper depths corresponding to the $\mathrm{CeO}_{2}$ buffer layer and the substrate. At these sample depths, the $\mathrm{S}$ vs. $E_{\mathrm{p}}$ curves for the two samples match, reaching ultimately the value of the Si substrate at $E_{\mathrm{p}}$ of $20 \mathrm{keV}$. Thus, the most noticeable features of $\mathrm{S}$ vs. $E_{\mathrm{p}}$ curves correspond to the Ge-region of the trilayer system.

Fig. 2 shows the results of the Raman measurements carried out on the as-grown $\mathrm{Ge} / \mathrm{CeO}_{2}$ film and sample annealed at $773 \mathrm{~K}$. As seen from Fig. 2a, the as-grown film exhibits a broad band with a maximum at approximately $274 \mathrm{~cm}^{-1}$, which is the characteristic feature of amorphous Ge [13]. However, for the annealed sample (Fig. 2b), a sharp Raman peak is observed at 303 $\mathrm{cm}^{-1}$, which is a clear signature of crystalline Ge [6]. The measured full width at half maximum (FWHM) of the sharp Raman line is $6.5 \mathrm{~cm}^{-1}$. Based on the correlated evidence from the two measurements (Figs. 1 and 2), it may be concluded that the top Ge layer of the as-grown film is amorphous containing a large density of open volume defects. This explains the pronounced peak in the S-parameter of the Ge-region. It is interesting that a similar peak in the S-parameter has been observed [14] earlier corresponding to amorphous Ge overlayers grown by thermal evaporation on GaAs substrate. The native defects of amorphous $\mathrm{Ge}$ get annealed at $773 \mathrm{~K}$, as revealed by the marked reduction in S-parameter corresponding to the Ge-region and complete crystallinity is recovered, as evident from the sharp Raman line in Fig. 2.

A positron diffusion model analysis of the experimental LEPB data has been made using VEPFIT code [15] to delineate the contributions to the S-parameter corresponding to the surface, epithermal, defect and substrate annihilation modes. The solid line through the data points is the result of VEPFIT analysis. The resolved defect lineshape parameter $S_{\mathrm{d}}$ for the Ge-region of asgrown film, normalised to $S_{\mathrm{B}}$ (the value for bulk $\mathrm{Si}$ substrate) is $S_{\mathrm{d}} / S_{\mathrm{B}}=1.017 \pm 0.001$. S-parameter measurements, using positron beam, have been reported [16] for hydrogenated amorphous $\mathrm{Ge}$ (a-Ge:H) films, addressed to the effect of ion-bombardment on the formation of vacancy structures. In this study, normalised S-parameter values in the range of 1.006-1.0250 have been observed for amorphous Ge films depending upon the hydrogen content, Ar-ion bombardment and substrate temperature. The larger S-parameter values were attributed to the formation of voids in the ion bombarded a-Ge:H film, while a lower S-parameter value of $\sim 1.016$ was assigned to the existence of atomic vacancies in the film without ion bombardment [16].

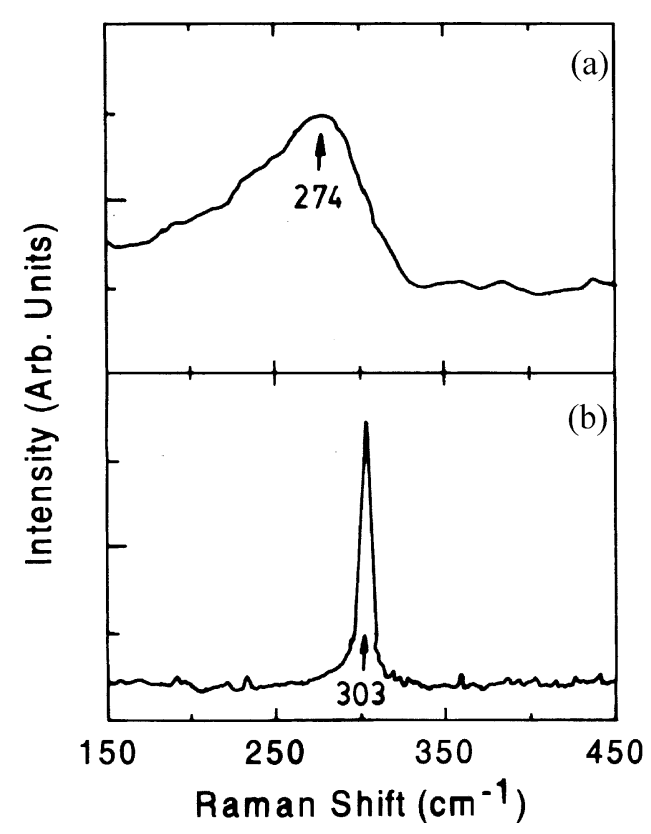

Fig. 2. Raman spectra obtained for (a) as-grown $\mathrm{Ge} / \mathrm{CeO}_{2}$ film and (b) film annealed at $773 \mathrm{~K}$. 


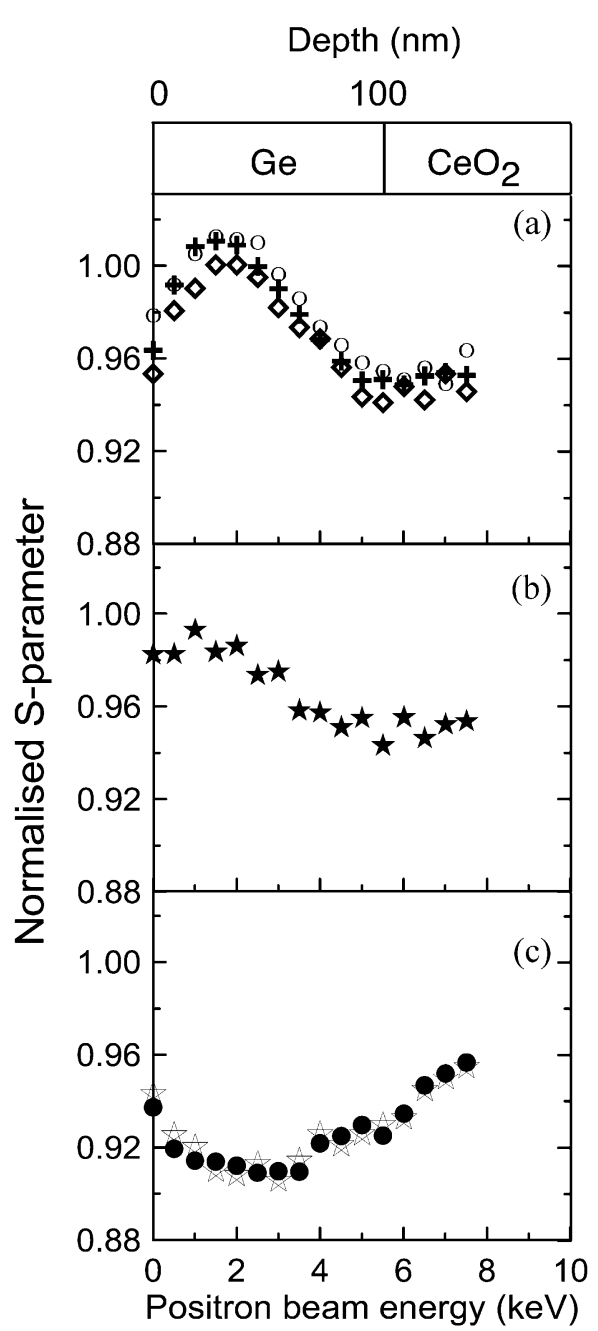

Fig. 3. Evolution of the depth profiles of the normalised S-parameter across the Ge-region for different annealing temperatures. For the sake of clarity, the different depth profiles are shown in three panels. (a) as-grown film $(\bigcirc)$; $373 \mathrm{~K}(+)$; $473 \mathrm{~K}(\diamond)$. (b) $573 \mathrm{~K}(\star)$. (c) 673 $\mathrm{K}($ 㳄) and $773 \mathrm{~K}(\mathbf{O})$.

The resolved S-parameter value $(1.017 \pm 0.001)$ in the present study matches well with the above reported experimental value for vacancies, thereby indicating that the amorphous state of as-grown Ge film contains a large concentration of open-volume defects, whose average size is of the order of monovacancy. Also, a comparison of the present results with those on a-Ge:H films [16] seems to suggest the combined role of hydrogenation and ion-bombardment in stabilising nanovoids in amorphous Ge films.

The transition from amorphous to crystalline Ge has been investigated through successive isochronal annealing measurements of the depth resolved S-parameter in the temperature range of 300-773 K. Fig. 3 shows the evolution of the depth profile of the normalised Sparameter across the Ge-region with the annealing temperature. S-parameter normalisation is the same as that of Fig. 1. The peak in S-parameter seen at approximately $E_{\mathrm{p}}$ of $2 \mathrm{keV}$ for the as-grown film stays for the $373 \mathrm{~K}$ and $473 \mathrm{~K}$ annealed samples. These profiles with similar features are shown in Fig. 3a. For the case of the 573 $\mathrm{K}$ annealed sample, the peak in the S-parameter becomes smeared as seen in Fig. 3b. Near-flat behaviour in the S-parameter across the Ge-region is seen for both 673 $\mathrm{K}$ and $773 \mathrm{~K}$ annealed samples and the profiles for the two cases match as shown in Fig. 3c. Fig. 4 shows the variation of the as-measured S-parameter, averaged over the depth interval corresponding to the Ge-region, as a function of annealing temperature. As against this variation for the Ge-region, the S-parameter corresponding to the buffer $\mathrm{CeO}_{2}$ layer and the $\mathrm{Si}$ substrate (not shown) remain constant as a function of annealing temperature. As seen in Fig. $4, \bar{S}_{\mathrm{Ge}}$ remains constant until $370 \mathrm{~K}$, beyond which it shows a monotonic decrease before levelling off at $T_{\mathrm{A}} \geq 673 \mathrm{~K}$. Detailed positron lifetime measurements have been reported [17] on single crystal Ge samples, irradiated to different electron doses. Because of the high dose of electron irradiation, a large disorder in terms of appreciable concentration of vacancies was found to be present, based on the observed lifetime parameters. It is interesting to note that a recovery stage at approximately 450 $\mathrm{K}$ was observed for electron doses of $10^{19} \mathrm{~cm}^{-2}$, which was ascribed to the annealing of either monovacancy or vacancy-oxygen complex. Notwithstanding the coarse annealing interval used in the present measurement, the observed S-parameter behaviour above $400 \mathrm{~K}$ is in broad agreement with the earlier reported result on vacancy annealing [17]. Based on the variation seen in Figs. 3 and 4, it may be qualitatively argued that above $400 \mathrm{~K}$, the onset of amorphous-to-crystalline transition is possibly initiated by the annealing of structural vacancies in the amorphous state, leading to the nucleation of

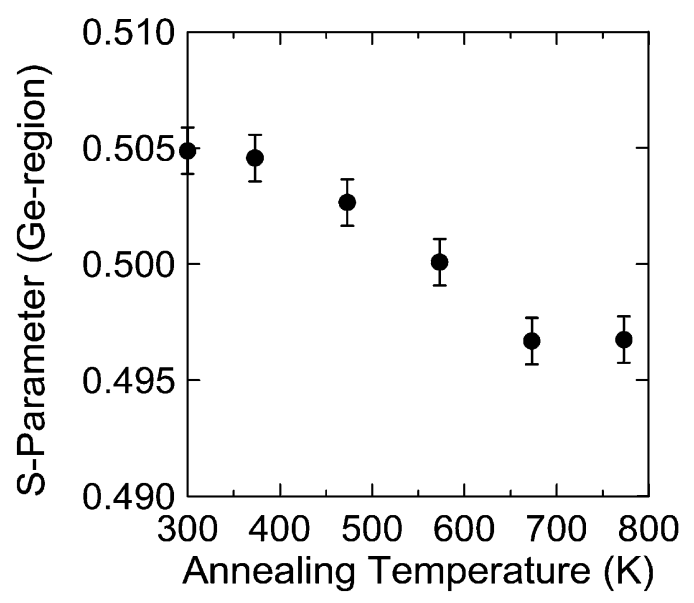

Fig. 4. Variation of the S-parameter, averaged over the depth interval corresponding to the Ge-region as a function of isochronal annealing temperature. 
microcrystalline $\mathrm{Ge}$ grains. In the partially crystallised state, there is a reduction in the number density of the native vacancy defects. At the same time there is a positive contribution to the defect density when a microcrystallite of $\mathrm{Ge}$ and an amorphous fraction coexist in the $\mathrm{Ge} / \mathrm{CeO}_{2}$ system. This arises from the compressive strain due to lattice mismatch between Ge (lattice constant $=5.64 \AA$ ) and $\mathrm{CeO}_{2}$ (lattice constant $=5.41 \AA$ ), which is as much as $4.5 \%$. The overall defect density is responsible for the intermediate state observed in the Sparameter behaviour at approximately $570 \mathrm{~K}$. At higher annealing temperatures, the microcrystallite size increases, resulting in a further reduction of the number density of defects and hence leading to a further decrease of the S-parameter. Complete crystallinity of Ge, free of defects is restored at $T_{\mathrm{A}} \geq 673 \mathrm{~K}$, as indicated by the flat behaviour of depth profiles seen in Fig. $3 \mathrm{c}$ and the constant values reached in Fig. 4. The present trend is also consistent with the observation of defect-free lifetime values beyond the annealing temperature of $625 \mathrm{~K}$ in electron irradiated Ge sample [17]. Thermal annealing results on Ge microcrystals embedded in $\mathrm{SiO}_{2}$ [18] have shown more blue shift for smaller microcrystals and the magnitude of this blue shift was found reduced with the increase of crystallite size. The temperature dependence of the incubation time for amorphous Ge films between 420 and $770 \mathrm{~K}$, studied by means of in-situ transmission electron microscopy and Raman spectroscopy [19], has indicated a crystallisation process involving nucleation and growth. The present annealing results on the amorphous-to-crystalline transition are in broad agreement with these earlier results.

\section{Summary}

The results of depth resolved positron beam and Raman spectroscopy measurements on a $\mathrm{Ge} / \mathrm{CeO}_{2}$ system deposited on a $\mathrm{Si}(100)$ substrate are reported. Both the measurements show correlated evidence that the asgrown Ge layer is amorphous, with a large concentration of structural vacancies. The isochronal annealing results of line shape parameter indicate amorphous-to-crystalline transition in the Ge film, possibly initiated by the annealing of structural vacancies. Complete crystallinity in terms of defect-free state is restored at $773 \mathrm{~K}$, as evident from both Raman and positron beam measurements.

\section{Acknowledgments}

One of the authors (A.K.S.) thanks the Department of Science and Technology for financial assistance.

\section{References}

[1] R.J. Nemanich, C.C. Tsai, G.A.N. Connell, Phys. Rev. Lett. 44 (1980) 273.

[2] M. Cardona, G. Guntherodt, Light Scattering in Solids, 5, Springer, Berlin, 1989.

[3] J. Fortner, R.Q. Yu, J.S. Lannin, Phys. Rev. B 42 (1990) 7610.

[4] S. Kanakaraju, S. Mohan, A.K. Sood, Thin Solid Films 305 (1997) 191.

[5] S. Kanakaraju, A.K. Sood, S. Mohan, Curr. Sci. 74 (1998) 322.

[6] S. Kanakaraju, A.K. Sood, S. Mohan, J. Appl. Phys. 84 (1998) 5756.

[7] S. Kanakaraju, A.K. Sood, S. Mohan, Phys. Rev. B 61 (2000) 8334.

[8] W. Brandt, A. Dupasquier, Positron Solid State Physics, NorthHolland, Amsterdam, 1983.

[9] P.J. Schultz, K.G. Lynn, Rev. Mod. Phys. 60 (1988) 701.

[10] G. Amarendra, G. Venugopal Rao, A.K. Arora, K.G.M. Nair, T.R. Ravindran, K. Sekar, B. Sundarvel, B. Viswanathan, J. Phys. Condens. Matter 11 (1999) 5875.

[11] B.M. Arora, K.S. Chandrasekaran, M.R. Gokhale, G. Geeta Nair, G. Venugopal Rao, G. Amarendra, B. Viswanathan, J. Appl. Phys. 87 (2000) 8444.

[12] G. Amarendra, B. Viswanathan, G. Venugopal Rao, J. Parimala, B. Purniah, Curr. Sci. 73 (1997) 409.

[13] Y. Sasaki, C. Horie, Phys. Rev. B 47 (1993) 3811.

[14] A. Halec, P. Maguire, P.J. Simpson, P.J. Schultz, G.C. Aers, T.E. Jackman, P. Marshall, in: E. Ottewitte, A.H. Weiss (Eds.), Slow Positron Beam Techniques for Solids and Surfaces, AIP Conference Proceedings, 303, American Institute of Physics, New York, 1994, p. 53.

[15] A. Van Veen, H. Schut, J. de Vries, R.A. Hakvoort, M.R. Ijpma, in: P.J. Schultz, G.R. Massoumi, P.J. Simpson (Eds.), Slow Positron Beams for Solids and Surfaces, American Institute of Physics, New York, 1990, p. 171.

[16] Z.L. Peng, D. Comedi, F. Dondeo, I. Chambouleyron, P.J. Simpson, P. Mascher, Physica B 272-274 (1999) 579.

[17] A. Polity, F. Rudolf, Phys. Rev. B 59 (1999) 10025.

[18] M. Fuji, S. Hayhashi, K. Yamamotto, Jpn. J. Appl. Phys. 20 (1991) 687.

[19] F. Edelman, Y. Komem, M. Bendayan, R. Beserman, J. Appl. Phys. 72 (1992) 5153. 